POLLACK PERIODICA

An International Journal for Engineering and Information Sciences

DOI: $10.1556 / 606.2017 .12 .3 .6$

Vol. 12, No. 3, pp. 55-68 (2017)

www.akademiai.com

\title{
ANALYSIS OF PLATE GLASS COLUMNS: THE INFLUENCE OF FIXING AND THE COUPLING PARAMETER
}

\author{
${ }^{1}$ Andras JAKAB, ${ }^{2}$ Salem G. NEHME \\ Department of Construction Materials and Technologies, Budapest University of Technology \\ and Economics, Múegyetem rkp. 3, H-1111 Budapest, Hungary \\ e-mail: ${ }^{1}$ jakab.andras@epito.bme.hu, ${ }^{2}$ sgnehme@yahoo.com
}

Received 31 December 2016; accepted 21 June 2017

\begin{abstract}
The design methods of the load bearing glass columns developed extremely in the last years. Numerous glass researchers have been trying to optimize the glass structures in terms of cost-effectiveness and safety. The authors carried out several laboratory experiments to analyze the key factors of the glass column design. Factors are investigated in the present article for example the influence of the fixing at the end of the glass columns, which essentially modifies the load bearing capacity of structures. The efficiency of the gluing of the interlayer foil (the coupling parameter) was analyzed based on the individual laboratory experiments.
\end{abstract}

Keywords: Glass, Columns, Stiffness of fixing, Coupling parameter, Stability

\section{Introduction}

The glass designing methods develop year after year. There are several difficulties concerning the designing of the viscoelastic laminated glass material. The stress distribution between two glass layers depends significantly on the interlayer foil. Nowadays, the residual capacity of the glass structures - after the fracture of the glass layers - increased and became predictable and can be designed as well thanks to the newer generations of the interlayer foils.

In the present paper, the stability of the plate glass columns and their designing parameters are investigated for instance: the fixing stiffness and the coupling parameter, which enables the calculation of the stress sharing between laminated glass layers.

The glass modeling is a popular topic today. Earlier Andreas Luible worked on the stability of the glass structures and defined possible methods for the designing [1], [2]. 
Feldmann and Langosch created buckling curves for glasses and they designed glass columns with the application of the well-known characteristic of buckling curves [3]. More complex statistical (Weibull) analysis appear to treat the strength, the loading and the probability of failure in one statistical algorithm for more safe and easier glass design [4].

Chiara Bedon and Claudio Amadio investigated a long time ago the possibility of design of glass structures with application of finite element models and laboratory experiments [5], [6], [7]. Roman Kalamar et al. investigated the load bearing capacity of glass hollow box columns [8].

Various problems were discussed in this complex research. First of all, the fracture pattern was investigated [9]. The load bearing ratios of the plate glasses with different thicknesses were investigated [10]. A method was invented for the buckling load determination; in general the moment of buckling does not occur at a well-defined specific load level based on the loading histories [11].

After numerous laboratory experiments of the authors, different types of buckling and stages were observed in the loading histories [12]. The following stages were observed based on the laboratory results. The loading history of the specimens started with a stable stage. In this stage the loading force increased with the vertical displacement without significant horizontal displacement. The loading force reaches a specific point where the specimen loses its stability, this is a short stage, which is called the service limit state and this point is also named the indifferent point in the stability theory. After the moment of buckling, the second stable stage starts and it ends at the fracture moment.

During the analysis of the loading histories of the specimens it was revealed that some characteristic stages were missing. According to the missing stages the specimens could be divided into different groups which are called buckling groups (Fig. 1). The first group contains those specimens, which have all of the mentioned stages; however, the first stable stage extends in the post-critical range without horizontal displacement. At the moment of buckling, the loss of stability is accompanied by rapid lateral movement and fast force decrease.

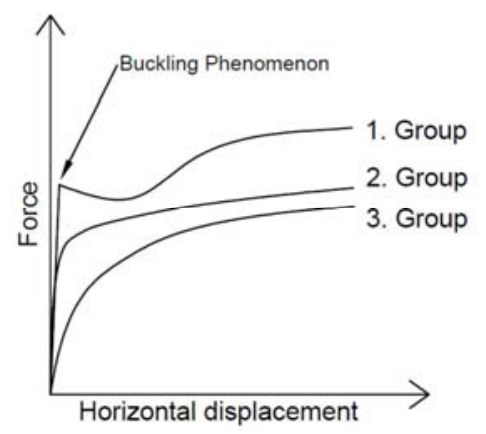

Fig. 1. Loading histories of the buckling groups 
The buckling force is sometimes higher than the force of the fracture, therefore this type of buckling is dangerous. For instance: when the overloaded glass column (which belongs to the first group and it is after the indifferent point in the first stable stage) gets a lateral impact, it breaks directly endangering the overall stability of the building.

In case of the second buckling group, the well-defined indifferent point disappeared. It is difficult to estimate the buckling moment load. However, the observed service limit state is rather predictable in the loading histories of the second group than in the first buckling group. This type of buckling group has enough resistance, so it can be used as load bearing element and it is safe enough. The third buckling group contains those specimens where only one stable stage can be observed due to the higher initial imperfection in the shape. The third is the safest buckling group, because the service limit state is on very low level; however it is not efficient at the utilization of the load bearing capacity. The buckling groups were observed in case of small-scaled specimens, where the incidence rate was determined. Probably the number of the specimen of the third buckling group increases in case of real plate glass columns due to the higher initial imperfection.

The reasons of the grouping were analyzed after the determination of the buckling groups, where different influencing factors were nominated [13]. The manufacturing procedure and the initial imperfections were under investigation in the last articles and in the present article different influencing factors of the buckling phenomenon are introduced.

The glass has to be strengthened with new layers and with new technologies, so the CFRP between the glass layers is successfully applied at the conventional building materials [14]. The applying of the newer, stiffer interlayer foils means at least twice of the load bearing capacity of the glass columns, if the general polymer: polyvinyl butyral and the new technology the Sentryglas plus interlayer foils are compared according to Qiang Liu et al. [15].

\section{Laboratory test set-up}

The goals of authors were to approach the buckling effect from the safety and to investigate the real behavior of the glass columns. Therefore, laboratory experiments were carried out in order to study the buckling behavior of single and laminated glass columns. Specimens were examined with the application of Instron 5989 testing machine. The scales of the geometry of specimens (height, thickness, width) were selected based on existing glass columns from international and Hungarian realized projects. Test parameters of glass specimens were selected as the following: Constants: test arrangement; support type; glass width $(80 \mathrm{~mm})$; interlayer material (ethyl vinyl acetate (EVA) foil with thickness of $0.38 \mathrm{~mm}$ ); edgework with polished edges; temperature $\left(+23 \pm 5{ }^{\circ} \mathrm{C}\right)$. Variables: type of glass layers; Heat Strengthened Glass (HSG)/non heat-treated float glass (annealed glass); height of specimens: $1000 \mathrm{~mm}$, $920 \mathrm{~mm}, 840 \mathrm{~mm}$; number of glass layers and the thickness of specimens; single layer: $8 \mathrm{~mm}, 12 \mathrm{~mm}, 19 \mathrm{~mm}$; laminated: $4.4 \mathrm{~mm}, 6.6 \mathrm{~mm}, 8.4 \mathrm{~mm}, 8.8 \mathrm{~mm}, 10.10 \mathrm{~mm}$; laminated: $4.4 .4 \mathrm{~mm}$ (the '.' means the position of the interlayer foil in the laminated glass structure; e.g. 4.4.4 means a laminated glass structure which contains three glass 
layers and two layers of interlayer foil). The rate of loading: $0.5 \mathrm{~mm} / \mathrm{min}, 1 \mathrm{~mm} / \mathrm{min}$. Support: height of fixing: $95 \mathrm{~mm}$; rubber plate (Shore A 80) was used between the steel supports and the glass. Simplified designations are used to distinguish between the studied specimens, these are e.g. H_2(4.4)-2-920-0.5: H, F: Type of glass: H - HSG; F - non heat-treated float glass; 2(4.4): Number of glass layers ex. $4.4 \mathrm{~mm}$ laminated glass; 2: The number of specimen; 920: Nominate height of specimen $[\mathrm{mm}]$; 0.5: Rate of loading [ $\mathrm{mm} / \mathrm{min}]$.

Abbreviations were used for the float Laminated Glass (LG) and for heatstrengthened laminated glass (Laminated Safety Glass (LSG)). Although laminated glass with Poly Vinyl Butyral (PVB) interlayer foil and Fully Tempered Glass (FTG) were not the part of the test parameters, a few pieces of these were tested as well.

The load and vertical displacement of the upper cross-head of the Instron 5989 universal testing machine were continuously measured. At three different heights the buckling displacement (horizontal displacement) of all specimens were continuously measured with displacement transducers during the tests. Strains at center point on the surface of the glass panels were measured with HBM LY11-10/120 strain gauges. At least three specimens were tested at each testing combination. Laminated specimens were loaded until all glass layers were fractured.

More than 250 specimens were loaded until fracture, and several different examinations were carried out, therefore more than 300 tests can be calculated. The glass properties (for instance: curvature) can vary in case of the same type of glass, therefore in several cases the same specimens were loaded by perpendicular loading force to the glass surface before the main test. The main test was introduced in Fig. 2.
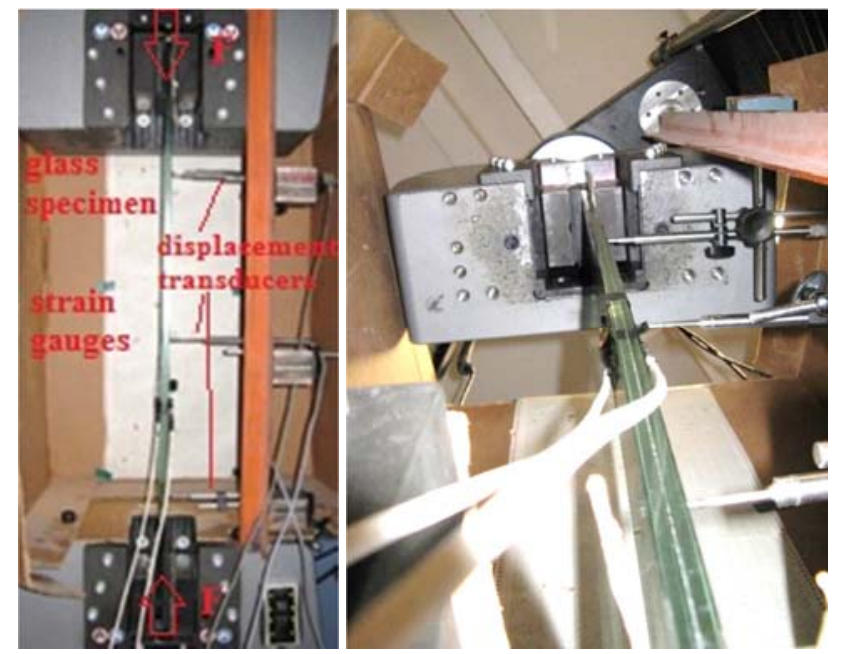

Fig. 2. The first laboratory test set-up and a buckling specimen

The load bearing capacity can be increased by the modification of the stiffness of the fixing in case of columns, especially glass columns. Another test set-up can be seen in Fig. 3. The analyzed glass specimen was built in the same position at this additional 
experiment. Strain gauges and displacement transducers were applied to measure more accurate data than the other test set-up. Partly pinned and partly fixed endings were applied to measure the different figures, deflections and rotations. The difference between the two test set-ups is the loading direction. Compression load cell is applied in the middle of the glass specimen in Fig. 3. Thin metal plates were applied between the glass surface and the compression load cell. According to the third Newton's law of motion, the measured force is the clear loading force that compresses horizontally the glass on the middle of the glass specimen. The applied supports at the end of specimens are real examples from buildings. At the general classical test set-ups that can be observed in different studies (e.g. at the hinge support [16]) the influence of the damping material is neglected, however each test set-up (damping material type, and thickness) effects different ways on the load bearing capacity of glass columns.

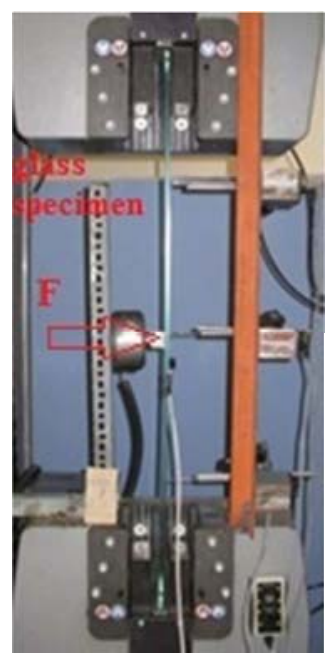

Fig. 3. The second laboratory test set-up to analyze the stiffness of the fixing

Stepwise loading was applied during laboratory experiments by the increase of the number of the thin metal plates. Photos were taken in every loading step to analyze the figure of the glass columns. The saving of the data of the load cell and the other measuring equipment were constant. The second test set-up or laboratory experiments were necessary to investigate the boundary conditions of the first test set-up. Four different glass specimens were tested by the second test arrangement, where the location of the concentrated load (middle and at the lower third) and the boundary conditions (partly pinned and partly fixed) were varied. These test set-ups are not an ideal arrangement, because the ideal case bears a significant difference in the boundary condition due to the absence of the damping material. These test setups approach rather the real states except of the sizes because it is a small scaled laboratory experiment. In the real glazing, thicker interlayer foils are applied between the glass layers due to the higher efficiency, however the specification of these laboratory experiments is the small 
scaling, and therefore the thickness of the interlayer foil was fixed $0.38 \mathrm{~mm}$. The results of the test setups are measured and investigated by authors.

\section{Approximation of the fixing factors}

\subsection{Rotation}

The rotation and the deflection of the specimen can provide information about the stiffness of the fixing. These results were investigated based on the second laboratory test set-up (Fig. 3), based on the results of four different glass specimens. This type of fixing is often applied at construction industry; therefore it is a real example. Two types of fixing were analyzed: partly pinned and partly fixed. Damping material has to be applied between the glass and steel material. Usually it is a kind of plastic, therefore maximal moment transmission cannot be created even in case of point fixing. When the place between the glass and steel is totally filled by rubber, it is called partly fixed because it is not perfectly fixed. Partly pinned fixing means that the tight steel shoe contains only one rubber layer. The glass column can rotate in the fixing freely, however, small amount of moment occurs due to the friction especially when the glass rotates more and the steel shoe supports it through the rubber. It means that less moment occurs. However, the rate of fixing is required to know that can be seen in the basic Euler formula in (1). The critical buckling force reacts sensitively to the modification of the fixing factor, which is signed by $v$.

$$
N_{c r}=\frac{\pi^{2} E I}{(v \cdot L)^{2}},
$$

where $N_{c r}$ is the critical bucking force [MPa]; $E$ is the Young modulus of the glass $[\mathrm{MPa}] ; I$ is the moment of inertia $\left[\mathrm{mm}^{4}\right] ; v$ is the Fixing factor $[-] ; L$ is the unsupported length of column [mm].

Different loading states can be compared to each other because of the applied stepwise loading. Photos were taken in each loading steps. The relative rotation of the glass specimen was compared via photos. Only one point of the specimen was compared to each other in aspect of the rotation in every loading steps at the middle of the fixed end (the height of fixing is $95 \mathrm{~mm}$ ), it means $47.5 \mathrm{~mm}$ from the end of the glass.

The measured force creates a moment at the fixing area. This real moment cannot be measured, therefore the maximal moment is signed, and this enables to theoretically analyze the differences between the two types of supports. Both slopes of lines should be less and the partly pinned still less in reality. Despite the fact that the moment is not real, this diagram is useful, because the ending moment cannot be approximated generally, therefore the theoretical maximal moment is also enough to calculate the rotation at the fixing. Fig. 4 introduces that the rotation is half of the partly fixed compared to the partly pinned support. 


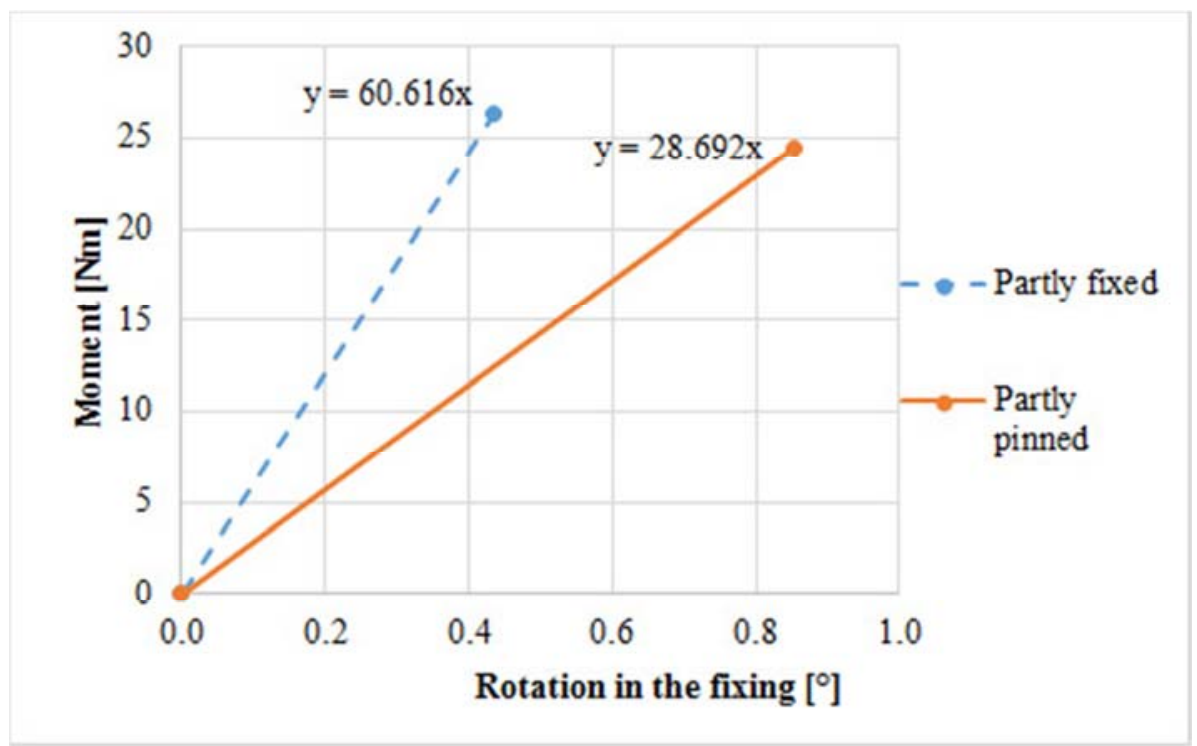

Fig. 4. Upper ending rotation in case of $\mathrm{H} \_2(10.10)$

Fig. 5 introduces the modification of the glass specimen rotation at the fixing in case of different glasses depending on the overall thicknesses. The rotation decreases if the inertia increases.

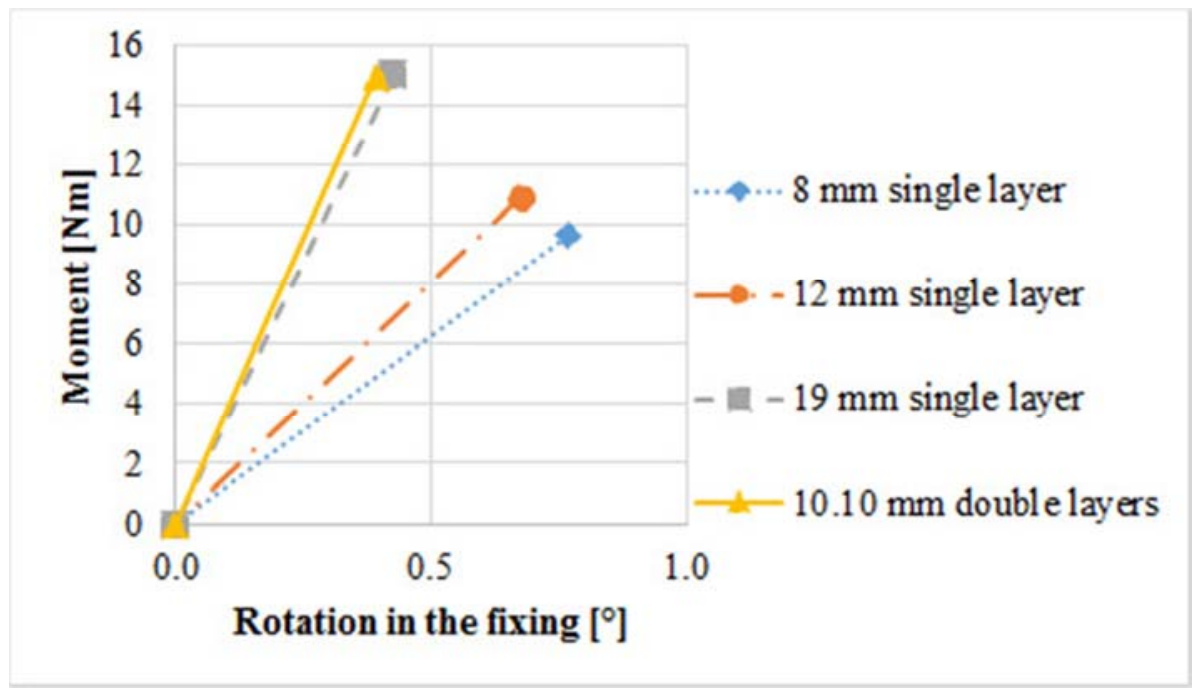

Fig. 5. Upper ending rotations in case of different overall thickness HSG (the number means in the marking the thickness of the specimen) 


\subsection{Deflection}

The stiffness of the fixing was analyzed based on the deflection data as well. The analyzed test set-up (Fig. 3) can be compared to a bended beam; therefore the elastic line differential equation can be applied. The basic formula is introduced in (2). The different factors of the differential equation are provided by the boundary conditions and the equations result the shear, the moment, the rotation and the deflection diagrams along the specimen depending on the type of fixing and the loading concentrated force.

$$
\frac{p_{z}(x)}{E I_{y}}=\frac{d^{4} w(x)}{d x^{4}},
$$

where $p_{z}(x)$ is the distributed load $[\mathrm{N} / \mathrm{m}] ; E$ is the Young modulus of the structure [MPa]; $I_{y}$ is the inertia of the structure $\left[\mathrm{mm}^{4}\right] ; w(x)$ is the deflection $[\mathrm{mm}]$.

The elastic line differential equations provide only the upper and the lower limit. The deflection for each point of the real structure was determined by vector graphic software from the photos, which were taken from the different steps of loading. The results of the real structure ought to be between the two limits. The experiments were carried out for several specimens, the deflection was determined in six cases along the specimen; each determined point was $20 \mathrm{~mm}$ from each other. The obtained deflection diagram belongs to the real specimen that could be compared to the limits.

In Fig. 6 the basic unloaded figure of the glass specimen (orange-circle), the loaded specimen (yellow-square), the real horizontal displacement (occurs the loading - redrhombus) and the two limits can be seen. The real figures provide information also about the sinus waves and overall bows; however this topic was submitted earlier [13]. Some sinus waves smooth out due to the loading. However, the measurement results also contain the uncertainty of the vector graphic procedure. The horizontal displacement is the difference between the loaded and unloaded states of the real specimen. The curve of horizontal displacement (red-rhombus) is compared to the two limits and its position is determined by linear interpolation for every measurement point. It does not mean that the obtained result is directly the fixing factor $(v)$, which is in (1). However, the effect of the damping material can be seen in this difference. The mean value of the linear interpolation is 0.71 . The sinus waves smooth out resulting in the roughness of the red curve and this phenomenon causes the higher differences.

In Fig. 7 the same specimen of Fig. 6 can be seen with partly pinned support. The horizontal displacement of the glass specimen is approximately equal to the lower limit aside from some small differences. The loaded specimen behaves as an almost perfectly pinned glass beam, where the fixing factor is 1.0. The mean value of the linear interpolation is above than 0.93 . It can be more than 0.98 if the result of the upper 5 measured points is neglected from the calculation of the mean. The ends of glass were not glue to the fixing steels, however these fixed joints can stiffen more the end stiffness and the efficiency of stiffness depends on the gluing material as well according to Machalicka et al. [17]. 


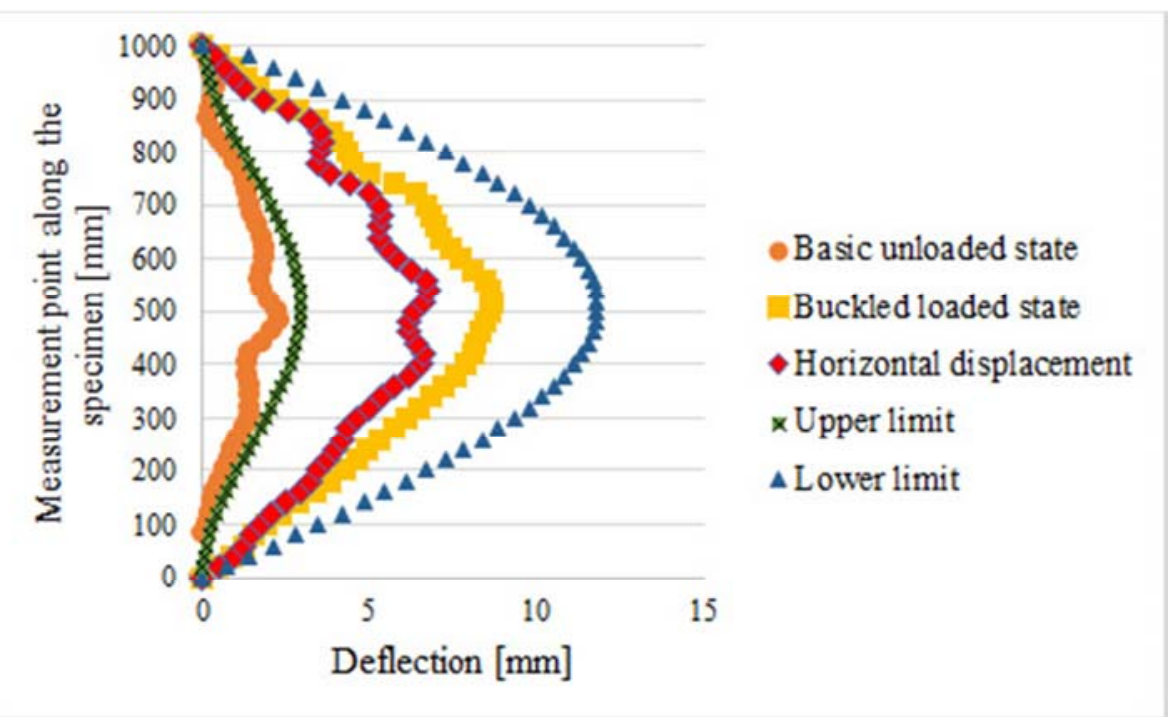

Fig. 6. Deflection measurement results of $8 \mathrm{~mm}$ thick glass specimen with partly fixed support

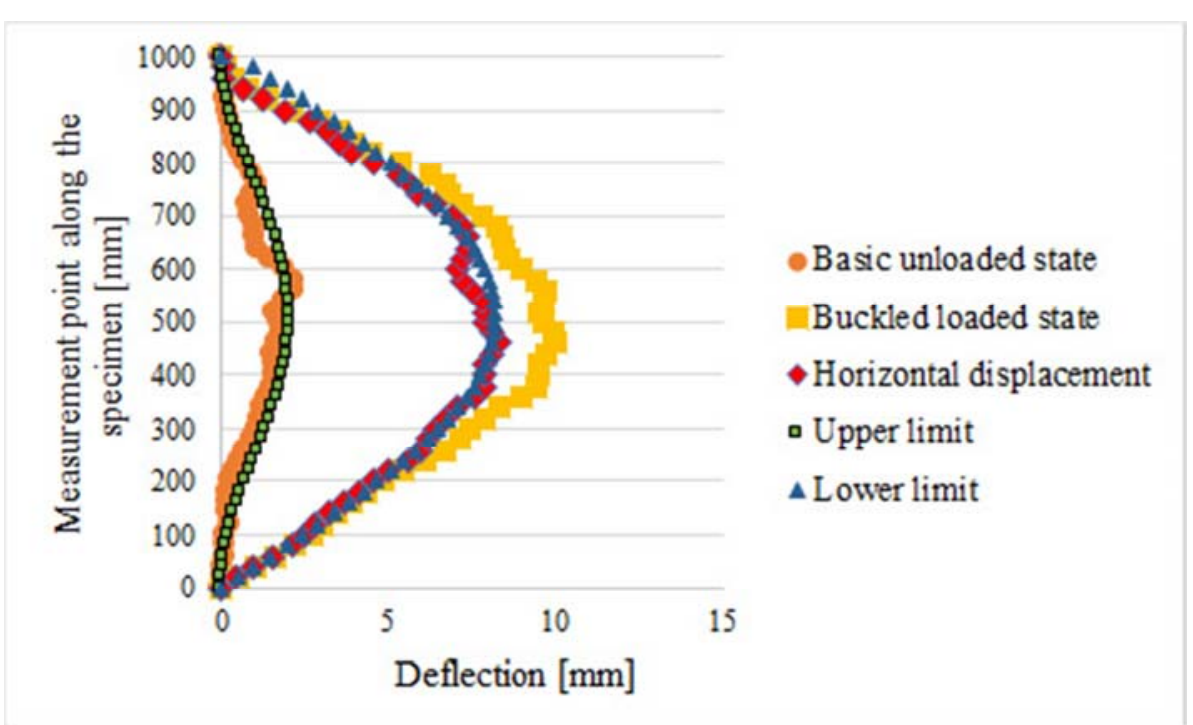

Fig. 7. Deflection measurement results of $8 \mathrm{~mm}$ thick glass specimen with partly pinned support

The critical buckling force of the vertical loaded structures depends on the buckling length and on how long sinus wave can be formed according to Euler. Therefore, the horizontal displacement shape of the partly fixed specimen was copied also into the vector graphical software, where the sinus wave length was determined. The result was 
0.76 which is 0.06 more than the interpolated version. These types of the fixing factor approximation ought to be validated by the general formulas from the literature.

The results of the second laboratory test set-up are required to provide data for analysis and behavior of the main buckling behavior. However, these small amounts of the additional tests are enough to demonstrate e.g. the boundary conditions. It is unnecessary to perform the additional tests (the second test set-up) for all specimens ( $250 \mathrm{pcs})$.

\section{Coupling parameter}

The stress distribution between two laminated glass layers is still an investigated topic nowadays. There are two limits: the monolithic and the lower layered limits and the real behavior are between these limits (Fig. 8). Thanks to the newer generation of interlayer foils, the stress distribution the laminated glasses approach the monolithic behavior, so the stress distribution is going to be maximal between the two layers. This principle is the near future of the glass designing.

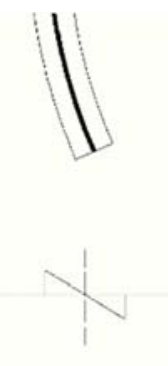

a)

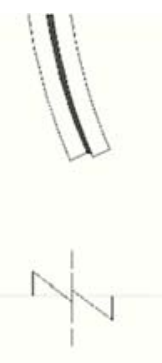

b)

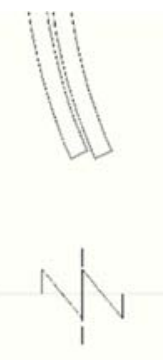

c)

Fig. 8. a) Monolithic behavior, b) real behavior; c) lower (layered) limit

J. Blauwendraad's approximation is the following [18]: there is a coupling parameter (contained in (3)) that is calculated by the flexibility of the layers (including the interlayer foils). The dimensionless $z$ parameter controls the contribution of the foil between the two glass layers. $N_{c r}$ is the critical buckling force (indifferent point). $L$ means lower limit; $U$ means upper limit in the indices. The coupling parameter is between $0-1$ values, it is 0 if the laminated glass consisted of non-bonded glass layers and $z=1$ in case of monolithically bonded glass layers

$$
N_{c r}=(1-\xi) N_{c r, L}+\xi N_{c r, U}
$$

An interesting experiment was carried out to analyze Blauwendraad's theory, based on the first introduced laboratory test set-up (Fig. 2). There were several loading histories from the basic tests, those results were introduced in [9], [10], [11]. However, the loading histories and the test results of the lower layered limit were missing. 
Therefore, 8 further glass pairs were loaded by concentrated axial load. Heat strengthened glasses and annealed glasses were tested. A comparison of the loading histories of four types of specimens is compared in Fig. 9, where the monolithic upper limit (blue-dashed line), the real behavior (laminated - black-continuous line) and the lower layered limit (red-dotted-dashed line and purple-dotted line) are indicated. There is no distance between the two individual glass layers in case of the red (red-dotteddashed line, it is marked 44) curve so during the loading the layers can bear friction. Rubber spacer is placed - about $5 \mathrm{~mm}$ thick - between two glass layers at the ends of the specimens $(10 \mathrm{~cm}$ in length from the top and from below, not along the whole specimens) in case of the purple (purple-dotted line, it is marked 4--4) curve. The effect of the friction can be seen if the red (red-dotted-dashed line) and the blue (purpledashed line) curves are compared, where the friction can increase the load bearing capacity slightly around the buckling load.

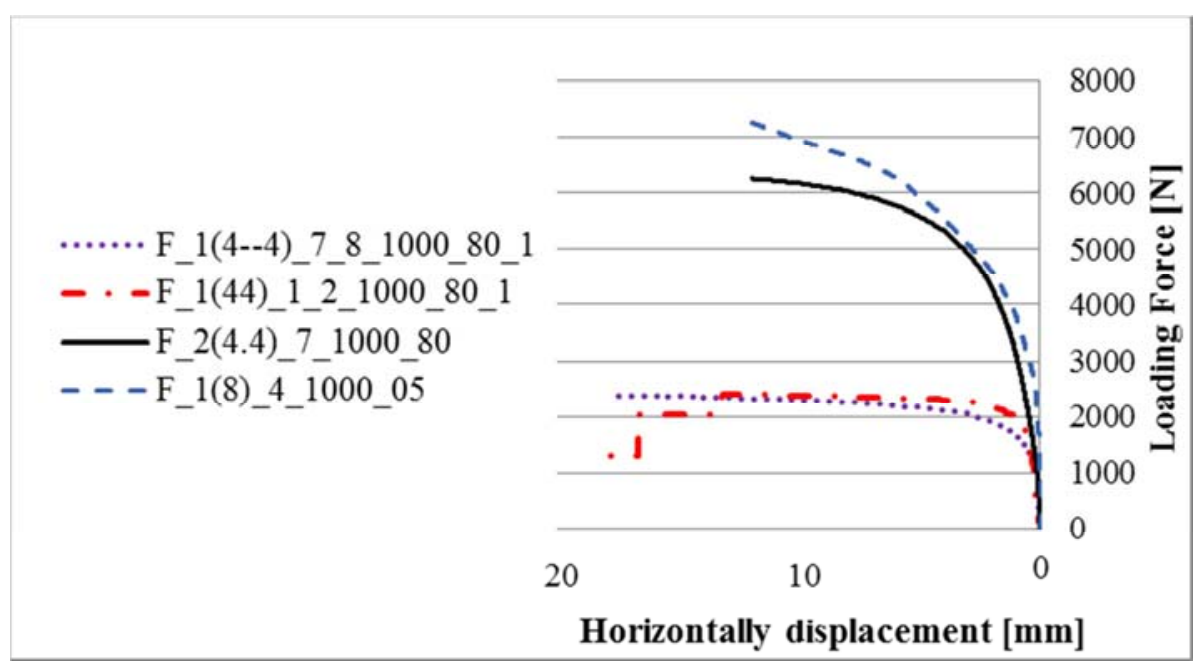

Fig. 9. Loading histories comparison

Blauwendraad defined his method only for the buckling load, however it is interesting if it can be applied for the fracture moment or for the whole loading history as well. It was already revealed that the heat strengthening procedure cannot increase significantly the critical buckling force; on the contrary, the procedure rather decreases that. The coupling parameter can be calculated only for well-defined moments, because the different glasses are not in the same states during the loading.

The characteristic forces of the buckling and fracture moment are compared to the upper limit in Table I, therefore 100\% value appears in the first raw. The percentages of the laminated glass and lower layered limit vary at the buckling load. However the values of the fracture moment decrease according to the expectations. The mentioned difference can be seen between the lower layered limit values. Where rubber is applied as spacer, the critical buckling force is lower, because there is no friction between the 
glass layers. The coupling parameter does not work properly at the buckling moment; however it works well at the fracture moment. The fracture moment of the lower layered limit can reach the third of upper limit that can be seen in the Fig. 9 and in the Table I. This ratio was observed in case of the heat strengthened glasses as well.

Table I

Comparison of the characteristic forces to the upper limit and the coupling parameter

\begin{tabular}{|c|c|c|c|c|}
\hline \multirow{2}{*}{ Limits and parameters } & \multicolumn{2}{|c|}{ The buckling load } & \multicolumn{2}{c|}{ The fracture moment } \\
\cline { 2 - 5 } & $\begin{array}{c}\text { Annealed } \\
\text { glass }\end{array}$ & HSG & Annealed glass & HSG \\
\hline Upper layered limit & $100 \%$ & $100 \%$ & $100 \%$ & $100 \%$ \\
Laminated glass 4.4 & $71 \%$ & $101 \%$ & $88 \%$ & $71 \%$ \\
Lower layered limit 44 & $76 \%$ & $63 \%$ & $37 \%$ & $32 \%$ \\
(4--4) & $(43 \%)$ & $(36 \%)$ & $(36 \%)$ & $(31 \%)$ \\
Coupling parameter 44 & $-22 \%$ & $104 \%$ & $80 \%$ & $58 \%$ \\
$(4--4)$ & $(50 \%)$ & $(102 \%)$ & $(81 \%)$ & $(58 \%)$ \\
\hline
\end{tabular}

\section{Conclusions}

The most important factors are the safety, the cost-effectiveness and the stability in today's glass designing. In the present paper, the Authors drew the attention to a kind of newer designer factors. From earlier studies, it is known that the fracture moment is independent of the buckling phenomenon and its force and displacement ranges move in narrow percentages, against of the buckling load.

The rotation of the end of glass specimen increases at least twice faster in case of the partly pinned glass support than a partly fixed support. The stiffening of the fixing is approached by elastic line differential equations, and the value of the fixing factor is determined as $0.71-0.76$. However, this result is not verified by the basic buckling formulas, it is going to be the continuation of the present article.

The coupling parameter does not increase obviously in case of HSG. The formula of coupling parameter provides more accurate results at the fracture moment. As it was shown, the fracture moment of the lower layered limit can reach the third of upper limit. This ratio was observed in case of the heat strengthened glasses as well.

Basic strengthening procedure of the glass column is going to be the developing of the interlayer foil. The calculation method has to be contained a general factor which depends on the properties of the foil to do the calculation as simple as possible, because the fast development of these foils is predicted.

The results of the tested glass specimens are not perfectly equal with the real sized glass columns, the size effect will be taken into the analysis in the further research.

\section{Acknowledgements}

The Authors express their gratitude to Rákosy Glass Ltd. for providing the specimens. Authors are thankful to the Department of Construction Materials and 
Technologies, Budapest University of Technology and Economics for the technical support. The rotation measuring is gratefully acknowledged to Melinda Réka Oláh and Alex Sinka civil engineer students.

\section{References}

[1] Luible A. Stabilitat von Tragelementen aus Glas, PhD Thesis 3014, Ecole Polytechnique Federale de Lausanne, Lausanne, 2004.

[2] Luible A. Plate buckling of glass panels, GPD 2005, Glass Processing Days Conference, Tampere, Finland, 17-20 June 2005, pp. 476-479.

[3] Feldmann M., Langosch K. Buckling resistance and buckling curves of pane-like glass columns with monolithic sections of heat strengthened and tempered glass, Challenging Glass 2, Conference on Architectural and Structural Applications of Glass, Delft, Netherland, 20-21 May 2010, pp. 319-330.

[4] Lamela-Rey M. J., Garcia-Prieto M. A., Fernandez-Canteli A. A probabilistic design model proposal for structural glass plates, Pollack Periodica, Vol. 1, No. 2, 2006, pp. 61-69.

[5] Amadio C., Bedon C. An analytical model for buckling evaluation of laminated glass beams in bending and compression, Proceedings of XXV A.T.I.V. International Conference: Glass, when Technology Meets Design, Parma, Italy, 18-19 November 2010.

[6] Bedon C., Amadio C. Buckling verification of laminated glass elements in compression, Journal of Civil Engineering and Science, Vol. 1, No. 3, 2012, pp. 90-101.

[7] Bedon C., Amadio C. Design buckling curves for glass columns and beams, Structures and Buildings, Vol. 168, No. 7, 2016 pp. 514-526.

[8] Kalamar R., Bedon C., Eliasova M. Experimental investigation for the structural performance assessment of square hollow glass columns, Engineering Structures, Vol. 113, 2016, pp. 1-15.

[9] Jakab A., Nehme K., Nehme S. G. Fracture behavior of glass columns, Experimental study of axial loaded glass columns, 3rd International Conference on Competitive Materials and Technology Processes, Miskolc-Lillafüred, Hungary, 6-10 October 2016, IOP Conf. Series: Materials Science and Engineering, Vol. 123, 2016, Paper No. 012056.

[10] Nehme K., Jakab A., Nehme S. G. Experiments on the buckling behavior of glass columns, Part 1, (in Hungarian) Építöanyag, Vol. 65, No. 4, 2013, pp. 112-117.

[11] Jakab A., Nehme K., Nehme S. G. Laboratory experiments of centrally loaded glass columns, GPD, Glass Performances Days Conference, Tampere, Finland, 24-26 June 2015, pp. 17-121.

[12] Jakab A., Nehme K., Nehme S.G. Stability questions of centrally loaded glass columns, METNET, The European Network of R\&D and Training Organisations of Metal Branch, Tenth International Annual Seminar, Budapest, Hungary, 13-14 October 2015, pp. 8-17.

[13] Jakab A., Nehme K., Nehme S. G. Classification of I shaped glass columns, CCC2016 Proceedings Creative Construction Conference, Budapest, Hungary, 25-28 June 2016, pp. $78-83$.

[14] Bedon Ch., Louter Ch. Finite-element numerical simulation of the bending performance of post-tensioned structural glass beams with adhesively bonded cfrp tendons, American Journal of Engineering and Applied Sciences, Vol. 9, No. 3, 2016, pp. 680-691.

[15] Qiang L., Xiaokun H., Gang L., Zhen Z., Gang L. Investigation on flexural buckling of laminated glass columns under axial compression, Engineering Structures, Vol. 133, 2017, pp. 14-23.

[16] Pesek O., Horacek M, Melcher J. Experimental verification of the buckling strength of structural glass columns, Procedia Engineering, Vol. 161, 2016, pp. 556-562. 
[17] Machalicka K., Eliasova M., Netusil M. Material properties of adhesives for shear bonded connections of structural glass, Pollack Periodica, Vol. 10, No. 2, 2015, pp. 57-68.

[18] Blaauwendraad J. Buckling of laminated glass columns, Heron, Vol. 52, Special No. 1-2, Structural glass, 2007, pp. 147-164. 